\title{
Naturally separated turbulent flow around two cylinders in tandem at high Reynolds number by time- resolved PIV.
}

\author{
M. ELHIMER, G. HARRAN, T. DELOZE, D. SZUBERT, Y.HOARAU, M. BRAZA \\ Institut de Mécanique des Fluides de Toulouse, UMR C.N.R.S.-I.N.P.T-UPS. 5502, Av. du Prof. Camille \\ Soula, 31400 Toulouse, France \\ melhimer@imft.fr
}

\begin{abstract}
In this study the focus is made on the properties of the bistable flow between two cylinder in tandem for a pitch ratio $L / D=3,7$ at a Reynolds number of 132800 . Time resolved particle image velocimetry (TRPIV) is used to measure the spatial distribution and time fluctuations of the velocity field in the wake of the upstream cylinder. From velocity spectra and statistics fields, the existence of two distinct seemingly stable flow patterns is shown, having different spatial structures and predominant frequencies.
\end{abstract}

Key words: Cylinders in tandem, time resolved PIV, bistable flow, turbulence.

\section{Introduction}

Multiple cylinder structures can be found in the designs for heat exchangers, cooling systems for nuclear power plants, offshore structures and cables, in both air and water flow. In many of these engineering applications, the periodic shedding of Kármán vortices is responsible for problems with flow-induced vibration and noise. Example of such cases is the triggering of fluid elastic instability in heat exchanger tubes arrays subjected to a cross-flow (Granger \& Paidoussis 1996) among other. The need to predict the critical velocity of this instability in the heat exchangers of nuclear reactors has motivated several experimental studies using PIV and visualization techniques (Paul 2007, Iwaki 2004) that show the complex interactions between the shear layer vortices and Kármán vortex streets in the flow field between the cylinders.

The tandem configuration is among the simplest form of multiple cylinders structure. The two cylinders are in close proximity, arranged at arbitrary centre-to-centre spacing and zero angle of incidence to the oncoming flow. This arrangement can be found in several locations on a landing gear, such as multiple wheels, axes, and hydraulic lines. It has been chosen by Jenkins et al. (2005) as a canonical problem to identify and assess the effect of the interaction of unsteady flow among components of the landing gear on the airframe noise generation on large aircraft during approach.

The tandem arrangement has shown a large variety of flow patterns depending on the pitch (e.g. the distance between the cylinders) to diameter ratio, 
the Reynolds number and the upstream turbulence intensity. These patterns have been investigated extensively by several authors (Igararashi 1981, Xu et Zhou 2004, Sumner 2000, Alam 2011, Lin et al. 2002) who show the existence of a critical pitch depending on the Reynolds number, concerning the transition from one pattern to another (Sumner 2010).

For the smallest values of the pitch to diameter ratio in the interval $(1,2)$, the tandem act as a single extended body because the downstream cylinder is located in the vortex formation zone of the upstream one, so this flow regime is called "extended body regime" in literature. The separated shear layers from the upstream cylinder wrap around the downstream cylinder, without any reattachment onto its surface, before rolling up alternately into Kármán vortices behind the downstream cylinder. The flow between the two cylinders is almost stagnant but may behave as a cavity flow (Sumner 2010).

For medium pitch ratio values in the interval $(2,5)$, the shear layers from the upstream cylinder reattach onto the downstream cylinder, with eddies formation and their shedding in the gap region between the two cylinders. A wide variety of fluid behaviours can be observed in this regime that was first classified by Igarashi (1981). In addition Lin et al (2002) show that the gap eddies can vary substantially and intermittently in terms of their strength, asymmetry, and general behavior.

Finally, at higher pitch ratios $L / D>5$ the downstream cylinder is sufficiently far away and therefore, a Von Kármán vortex shedding can occur past the upstream cylinder as well as past the downstream one. The downstream cylinder is located in this case outside the vortex formation region of the upstream cylinder and experiences the periodic impingement of shed vortices from the upstream cylinder. In this "co-shedding” flow regime, both cylinders undergo vortex shedding at the same frequency.

The transition to and from the co-shedding regime is known to be bistable, with intermittent appearance of the two flow patterns (Igarashi 1981, Xu et Zhou 2004). This bistable regime appears in several experiments with different aspects and blockage ratios (Sumner 2010). In the present study the focus is made on this bistable regime, the aim of our experiment is to fully characterize each of the two flow patterns. Time resolved PIV is used allowing resolving simultaneously the spatial structure and the spectral content of the velocity field in the wake of the upstream cylinder for each of the two configurations. A comparison between the properties of the two patterns is done. The present study shows that the bistable regime is observed for tandem cylinder with pitch to diameter $L / D=3,7 \times D$ at Reynolds numbers $\mathfrak{R}=107000$ and $\mathfrak{R}=132800$ but not in the lower Reynolds number range, ( $\Re=31000$ and 47000$)$. This suggests the existence of a critical Reynolds number beyond which the bistable regime appears. In this article the focus is made on the highest Reynolds number $\mathfrak{R}=132800$. 


\section{Experimental set-up}

The experiments were undertaken in the wind tunnel S4 of IMFT, which delivers an upstream velocity with low turbulent fluctuations (the turbulence intensity has been measured by means hot wire anemometry and found to be of order 0,15 ). The test section is of $58 \mathrm{~cm}$ width and $72 \mathrm{~cm}$ height. Two transparent Plexiglas cylinders of diameter $8 \mathrm{~cm}$ have been used, yielding an aspect ratio of 7,25 and a blockage ratio of 0,11 . This compares well with Lin et al. (2002) experiments were the aspect ratio is 8,3 and the blockage ratio was 0,1 . The aspect ratio and the blockage ratio were in Igarashi (1981) 4 and 0,06 respectively and in Xu et Zhou (2004) 40 and 0,025 respectively.

In the present study, the pitch to diameter ratio of the tandem is $L / D=3,7$ so the gap between the cylinders is $21,6 \mathrm{~cm}$ wide. The cylinders are positioned in cross-flow (figures 1 and 2) at the mid-height of the test section and spanned over its width.

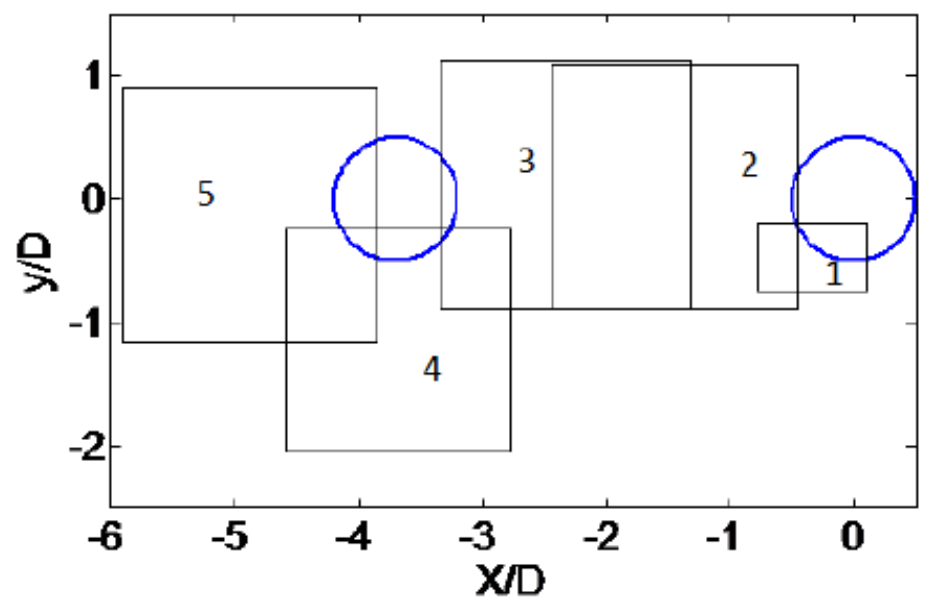

Figure 1 Measurement planes

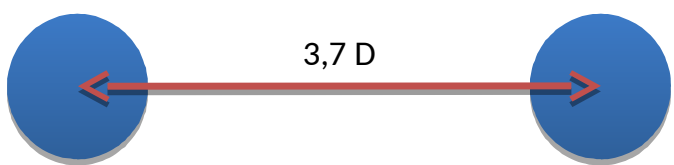

$\mathrm{U}=25,5 \mathrm{~m} / \mathrm{s}$

Figure 2 Tandem cylinder scheme and upstream velocity 
The upstream velocity in the wind tunnel was measured using a Pitot tube with pressure plugs at the inlet and the outlet of the contraction section, and was controlled by changing the fan angular velocity. The upstream velocity is $U=25,5 \mathrm{~m} / \mathrm{s}$ yielding a Reynolds number of $\mathfrak{R}=132800$.

The velocity field measurements have been performed using a high speed PIV set-up that includes a double frame high-speed camera (Photron RS3000), a twocavity Laser (Quantronix Darwin) and a synchronizer. The planes of measurement are shown in Fig. 1 concerning the whole data base measured. In the present study, the results of plane 2 are discussed.

The camera CMOS-sensors has a resolution of $1024 \times 1024$ pixels and an internal memory size of 8 GB enabling an acquisition duration of 3 seconds at a frequency of $2000 \mathrm{~Hz}$, the resulting PIV measurements frequency is $1000 \mathrm{~Hz}$. The visualization plane was located in the wake of the upstream cylinder at its mid-width with the size of $16 \mathrm{~cm} \times 16 \mathrm{~cm}$. In order to reach the statistical convergence up to the second order, ten PIV acquisitions were carried out for each of the two flow patterns: knowing that the frequency of the Von Kármán shedding is about $50 \mathrm{~Hz}$ in the present study, each acquisition contains 150 shedding cycles, thus about 1500 shedding cycles have been covered in our measurements for each pattern.

The energy of the laser beam delivered by each cavity was set at $25 \mathrm{~mJ}$. Using a combination of spherical and cylindrical lenses, the Laser beam was expanded into a light sheet of $2 \mathrm{~mm}$ thickness. The flow was seeded with droplets of a mixture of water and alcohol using a fog generator. The droplet size distribution was measured using a Malvern granulometer and found $3,4 \mu \mathrm{m}$ on average with a small standard deviation.

The image processing and the cross-correlation analysis were done by using DAVIS 8 La Vision software. The images intensity was inhomogeneous due to the lighting conditions and the sensor properties.

The high frequency of the PIV measurements allows extracting velocity temporal signal at any location of the visualization domain. For each location the time series from the ten acquisitions were concatenated in order to form a sufficiently long time series. The periodogram of these time series was computed using spectral averaging on multiple windows (Marple 1987) with nonoverlapping Hamming windows of size 512 samples, the resulting frequency resolution is $0,03 \mathrm{~Hz}$.

\section{Statistical and spectral analysis of the flow in the gap region between the two cylinders}

As mentioned above, for the pitch ratio $L / D=3,7 D$ and the Reynolds number $\mathfrak{R}=132800$, a random bifurcation has been observed concerning the gap flow between two flow patterns. The duration of each flow pattern is variable and it is generally of the order of several minutes, in comparison the Von-Kármán shedding period being of the order of 20 milliseconds. 
The first flow pattern, named I, consists on a Von Kármán vortex shedding in the wake of the upstream cylinder (Figure 1) but with a different shedding frequency: the dimensionless Strouhal number is $S t=0,178$ instead of $S t=0,21$ for a single cylinder. The second flow pattern, named II, appears generally after the first one. It consists of two stable detached shear layers that reattach on the downstream cylinder and create a recirculating flow in front of this cylinder (Figure 2). This pattern displays a Strouhal number of $S t=0,135$ close to the downstream cylinder.

The appearance of the flow pattern II always comes with $40 \mathrm{~Pa}$ increase of the pressure difference between the inlet and the outlet of the contraction section. Thus the upstream velocity increases by about $1 \mathrm{~m} / \mathrm{s}$ while the fan's angular velocity remains unchanged. This shows the decreases of the pressure drop induced by the presence of the tandem in the test section when the flow patterns II appears, and is probably due to the decrease of shear stress in comparison with the first pattern. In our experiments, the velocity was reduced after the appearance of the second pattern to bring it back to its initial value $U=25,5 \mathrm{~m} / \mathrm{s}$. The acquisition was started only two minutes later. This change of the upstream velocity does not cause the switching towards the first pattern.

\section{FLOW PATTERN I}

This pattern shows a topology and statistical properties similar to single cylinder in cross-flow. Indeed, The longitudinal average velocity field $U_{m}$ is symmetrical with respect to the axis $y / D=0$, and one can clearly identify the non-rotational zone, the shear zone and the dead water zone (Figure 3, Figure 5). In the irrrotational zone, the upstream flow is accelerated to $1,2 \times U$ near the cylinder and beyond the position $y / D= \pm 0,63$ in agrement with the results of Braza (2006) for an isolated cylinder at a close Reynolds number $\mathfrak{R}=140000$. In addition, the cylinder recirculating length, defined as the distance between the axis of the cylinder and the point were $U_{m}=0$, is $l_{c}=1,27 \times D$ which is in good agreement with the value $l_{c}=1,28 \times D$ obtained by Braza (2006). Finally, the Reynolds stresses fields (Figure 7, Figure 9, Figure 11, Figure 13, Figure 15, Figure 17,) shows a topology and non-dimensional values close to the case of an isolated cylinder.

The vertical velocity spectra have been measured at different positions on the line $y / D=0,5 \quad$ (Figure 19). They show a peak of power spectral density at the non-dimensional frequency $f . D / U=0,178$ thus defining the Strouhal number of the flow. This value is in good agreement with the results of $\mathrm{Xu}$ et Zhou (2004) and Igarashi (1981) for close pitch to diameter ratio but at smaller Reynolds numbers. As mentioned by Xu et Zhou (2004) the Strouhal number depends on both on the base pressure coefficient $C_{p b}$ and the shear layer thickness $\delta$

$$
S t=S t_{0}\left(1-C_{p b}\right)^{1 / 2} /(1+2 \delta / D) \quad \text { where } S t_{0} \approx 0.176 \text { is a universal Strouhal }
$$
number. The shear layer thickness depends on the Reynolds number and the base 
pressure coefficient is modified by the presence of the downstream cylinder. This explains the dependence of the Strouhal number on both the pitch and the flow Reynolds number.

For the same pitch to diameter ratio but at higher Reynolds number $\Re=166000$ Jenkins et al (2005) found a higher value of the Strouhal number $S t=0.26$. However their experiments are not comparable to ours because these authors tripped the first cylinder in order to force a turbulent boundary layer upstream separation.

\section{FLOW PATTERN II}

The flow pattern II has a distinct topology comparing to the case of isolated cylinder. The flow structure consists of two stable shear layers issued from the upstream cylinder. This observation is confirmed by examining the longitudinal average velocity field (Figure 4).

Two irrotational zones are identified at both sides of the upstream cylinder, were the mean longitudinal velocity reaches a higher value than in the first pattern: $1,3 \times U$ at $y / D= \pm 0,8$ on the line $x / D=0,71$. The shear zones located between $y / D= \pm 0,5$ and $y / D= \pm 0,8$ delimit a "dead water zone" that spans over a larger area than in the first flow pattern. Further away from the cylinder, the average longitudinal velocity becomes negative; this suggests the existence of a downstream recirculation flow that is however outside the visualization zone in these experiments. This recirculation is most probably generated by the reattachment of the shear layers on the downstream cylinder.

The normal Reynolds stresses have a symmetrical distribution with respect to the axis $y / D=0 \quad$ (Figure 8, Figure 12) while the Reynolds shear stress has an anti-symmetrical distribution with respect to this axis. The Reynolds stresses values are significantly smaller compared to the first flow pattern due to the attenuation of the organized large-scale flow close to the upstream cylinder.

The Reynolds stresses concentrate at the thickness of the shear zones that increase away from the cylinder due to the turbulent diffusion of the stresses. Indeed, the shear zone thickness increases from $0,25 D$ at $x / D=-0,71$ to

$0,5 D$ at $x / D=-1,5 D$ (Figure 10, Figure 14). The Reynolds stresses are negligible close to the cylinder in the "dead water zone" and increase as the recirculation flow get closer.

The vertical velocity spectra are shown along the line $y / D=0,5$ in this flow pattern (Figure 20). The spectrum is continuous near the cylinder because the large scale alternating motion is attenuated in this region of the flow in case of pattern II. However small vortex bursts are identified in the instantaneous PIV velocity fields in the shear zone. These vortices cannot be detected by a classical spectral analysis due to their high frequency appearance in respect of the sampling frequency of the TRPIV experiment ( $1000 \mathrm{~Hz}$ ).

For positions closer to the downstream cylinder a power spectral density (PSD) peak appears at the non-dimensional frequency $f . D / U=0,135$ that is different from the Strouhal number value of 0,178 in the first flow pattern. 
This value is however in good agreement with the findings of Xu et Zhou (2004) for close pitch to diameter ratio and at smaller Reynolds number. This peak is most probably linked to the recirculation flow in the front of the downstream cylinder and clearly appears beyond the position $x / D=-1,7$. This result indicates that the Von Kármán mode appears farther downstream and is characterized by a lower frequency, because of the vicinity with the second cylinder. The fact that this Strouhal number remains constant for these downstream positions associated with the presence of two inflexion points in the mean velocity profiles indicates that this mode is a Von Kármán mode.

\section{Conclusions}

In this paper, an experimental investigation of the flow between two cylinders in tandem with a pitch $L / D=3,7$ and a Reynolds number of $\mathfrak{R}=132800$ has been carried out. The separated shear layers from the upstream cylinder switch intermittently between roll-up with vortex shedding and reattachment onto the downstream cylinder with recirculation. The first flow pattern is similar to a Von Kármán shedding from an isolated cylinder but with a smaller Strouhal number. The recirculation is mainly outside our visualization domain but it is most probably responsible for the different value of the Strouhal number measured in the second flow pattern. Additional time-resolved PIV measurements have been undertaken, in order to fully characterize the recirculating flow and reattachment dynamics in the vicinity of the downstream cylinder, in comparison with simulation results for static and freely vibrating second cylinder.

\section{Acknowledgements}

This work has been carried out in the research group EMT2 (Ecoulements Monophasiques, Transitionnels et Turbulents) of the Institut de Mécanique des Fluides de Toulouse. It is based on the collaborative efforts of Sébastien Cazin, Moise Marshal, Hervé Ayroles and Christophe Korbuly for the experiments set up and the measurements techniques. Authors thank Alain Sevrain for useful discussions. This work has been carried out in the context of the ANRBARESAFE - "Simulation of Safety Bareer reliability", coordinated by EDF, Electricité de France.

\section{References}

Alam, Md. Mahbub, et J. P. Meyer. 2011. « Two interacting cylinders in cross flow ». Physical Review E

Braza, M., R. Perrin, et Y. Hoarau. 2006. « Turbulence properties in the cylinder wake at high Reynolds numbers ». Journal of Fluids and Structures

Granger, S., et M. P. Paidoussis. 1996. "An improvement to the quasi-steady model with application to cross-flow-induced vibration of tube arrays ». Journal of Fluid Mechanics

Igarashi, T. 1981. "Characteristics of the Flow Around Two Circular Cylinders Arranged in Tandem. I ». JSME International Journal Series B.

Iwaki, C., K. H. Cheong, H. Monji, et G. Matsui. 2004. «PIV Measurement of the 
Vertical Cross-flow Structure over Tube Bundles ». Experiments in Fluids 37

Jenkins, Luther, Mehdi Khorrami, Meelan Choudhari, et Cathy McGinley. 2005. "Characterization of Unsteady Flow Structures Around Tandem Cylinders for Component Interaction Studies in Airframe Noise ». American Institute of Aeronautics and Astronautics,

Lin, J.-C., Y. Yang, et D. Rockwell. 2002. "Flow past two cylinders in tandem: instantaneous and averaged flow structure ». Journal of Fluids and Structures

Marple, S. Lawrence. 1987. « Digital Spectral Analysis with Applications ». Englewood Cliffs, NJ, Prentice-Hall, Inc.

Paul, S.S., M.F. Tachie, et S.J. Ormiston. 2007. « Experimental study of turbulent crossflow in a staggered tube bundle using particle image velocimetry ». International Journal of Heat and Fluid Flow

Sumner, D., S. J. Price, et M. P. Paidoussis. 2000. « Flow-pattern identification for two staggered circular cylinders in cross-flow ». Journal of Fluid Mechanics

Sumner, D. 2010. « Two circular cylinders in cross-flow: A review ». Journal of Fluids and Structures

Xu, G., et Y. Zhou. 2004. "Strouhal Numbers in the Wake of Two Inline Cylinders ». Experiments in Fluids
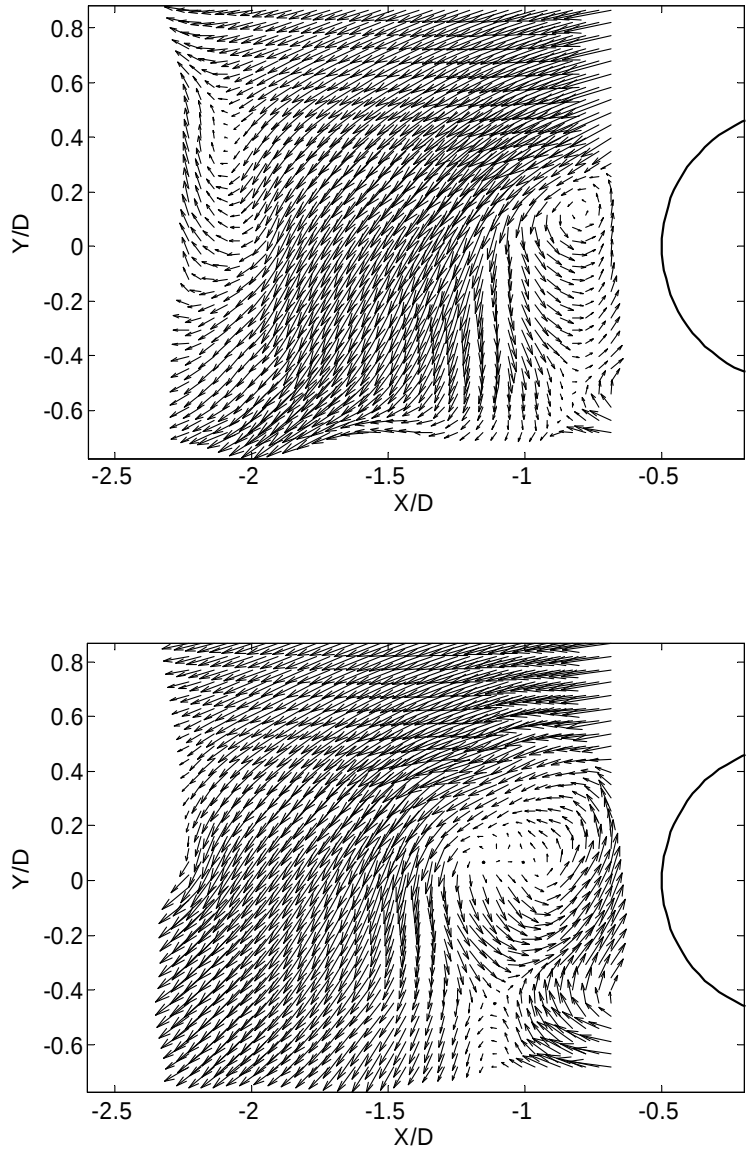
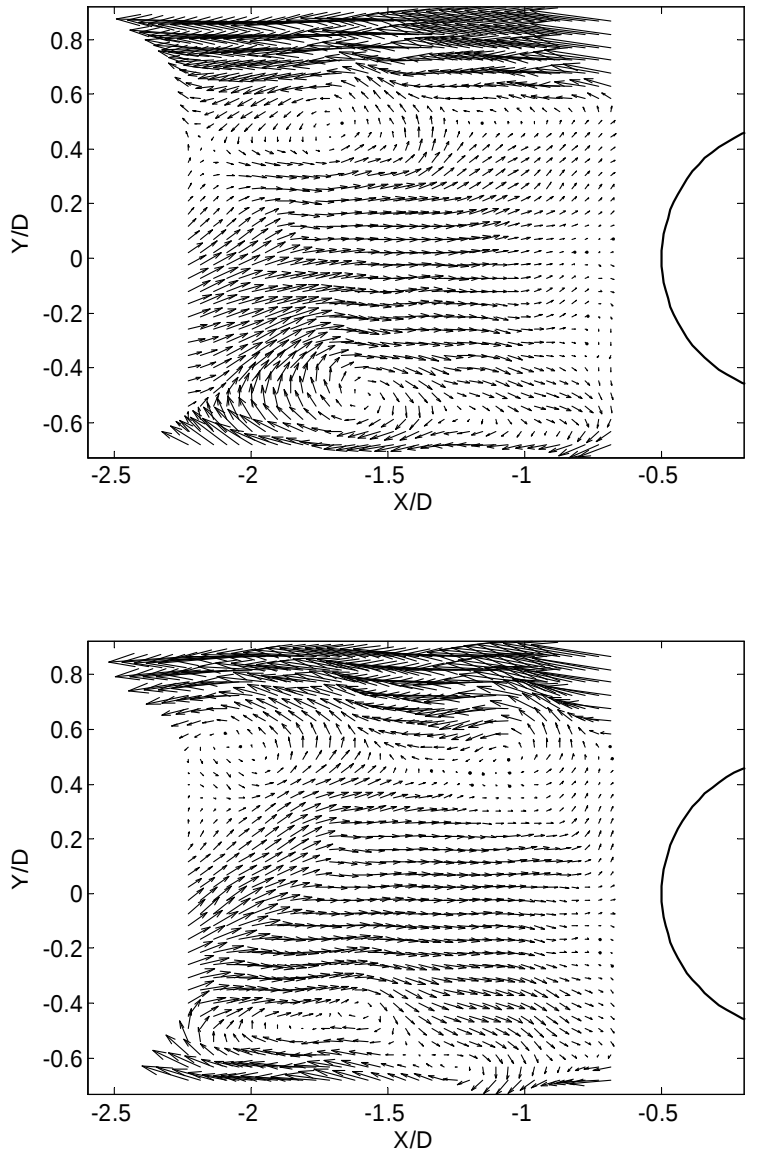

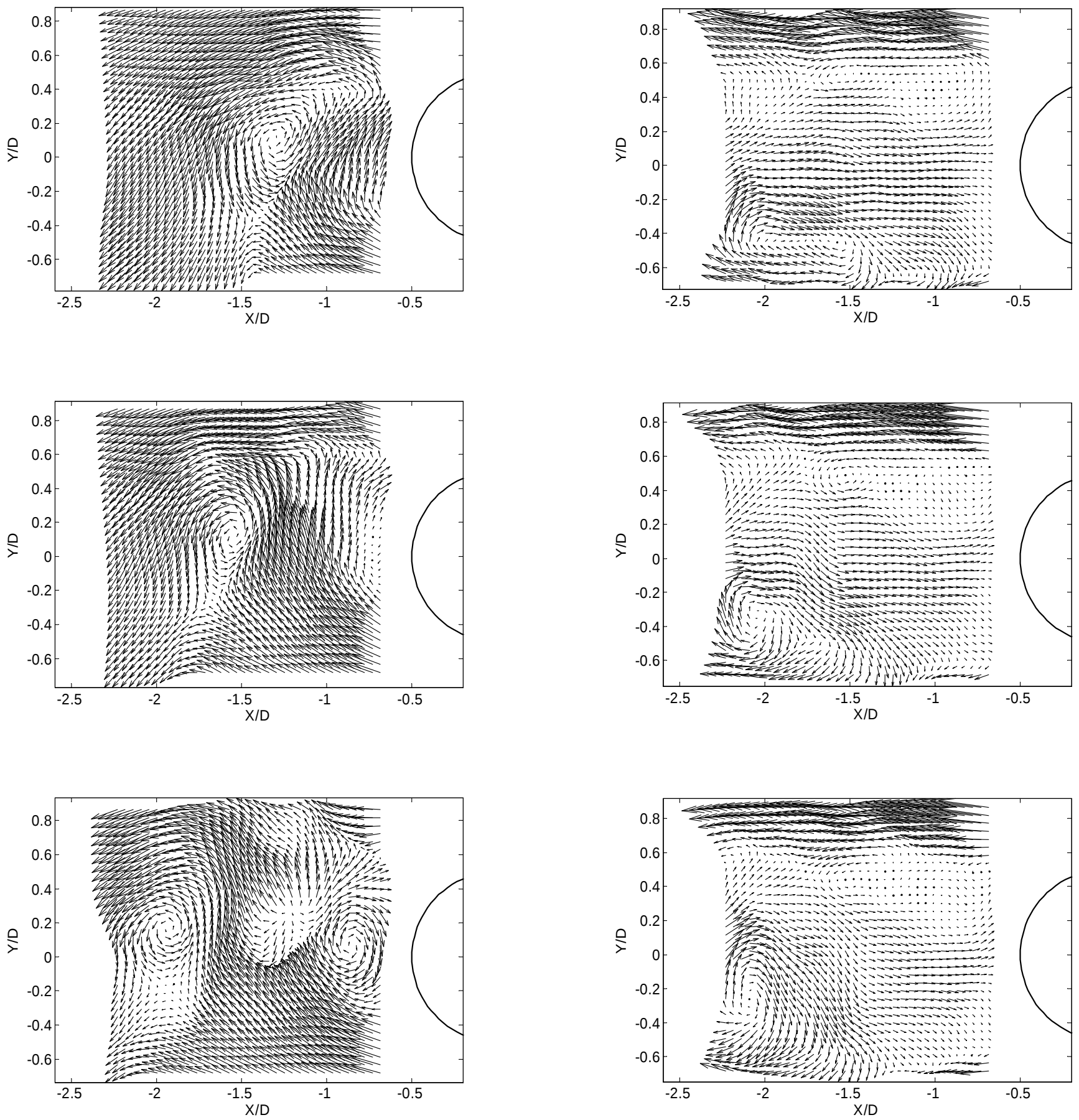

Figure 1 flow pattern I : Velocity field sequence at a frequency of $500 \mathrm{~Hz}$. The number of vectors have been reduced and the velocity fields smoothed for clarity
Figure 2 flow pattern II : Velocity field sequence at a frequency of $500 \mathrm{~Hz}$. The number of vectors has been reduced and the velocity fields smoothed for clarity 


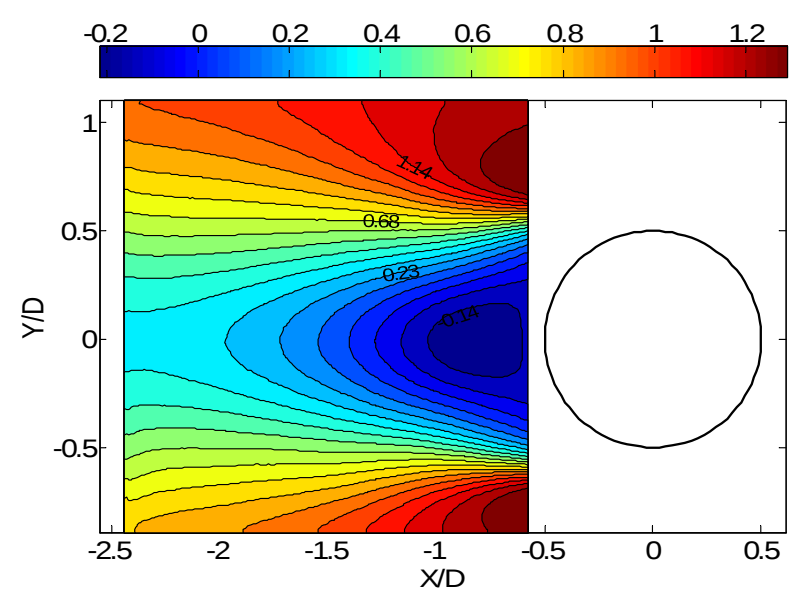

Figure 3 Flow pattern I : spatial field of $U_{m} / U$ in the wake of the upstream cylinder.

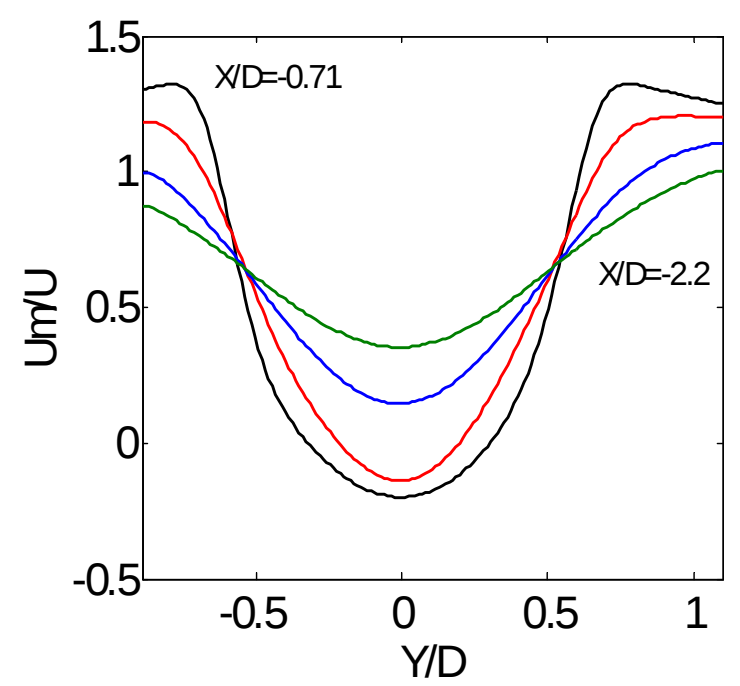

Figure 5 Flow pattern I : profiles of $U_{m} / U$ along the lines $\quad x / D=-0,71$ (black) $x / D=-1 \quad$ (red) $x / D=-1,5$ (blue) $x / D=-2,2 \quad$ (green).

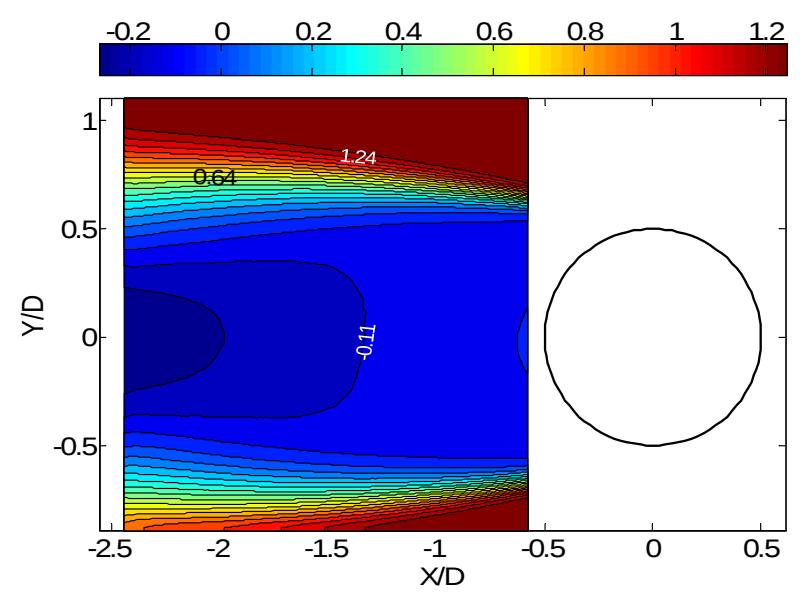

Figure 4 Flow pattern II : spatial field of $U_{m} / U$ in the wake of the upstream cylinder.

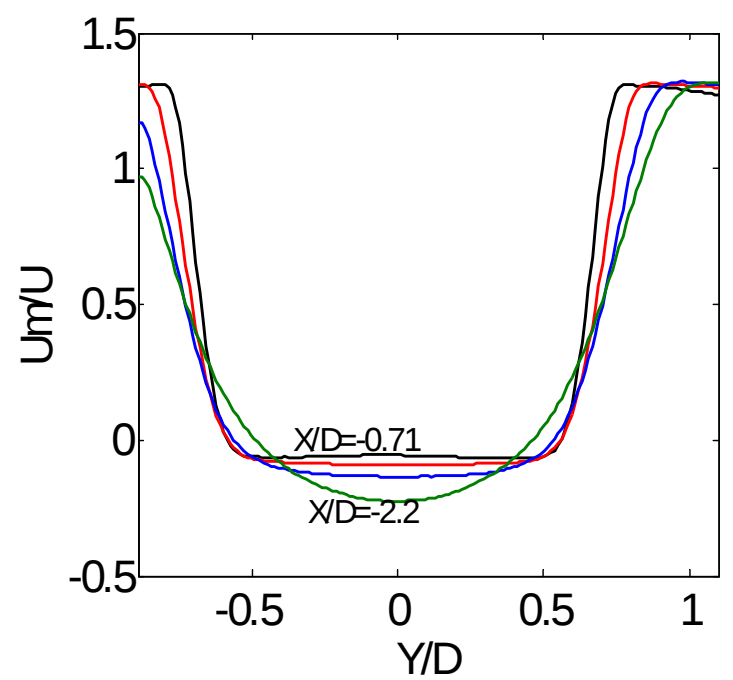

Figure 6 Flow pattern II : profiles of $U_{m} / U$ along the lines $\quad x / D=-0,71$ (black) $x / D=-1 \quad$ (red) $x / D=-1,5$ (blue) $x / D=-2,2 \quad$ (green). 


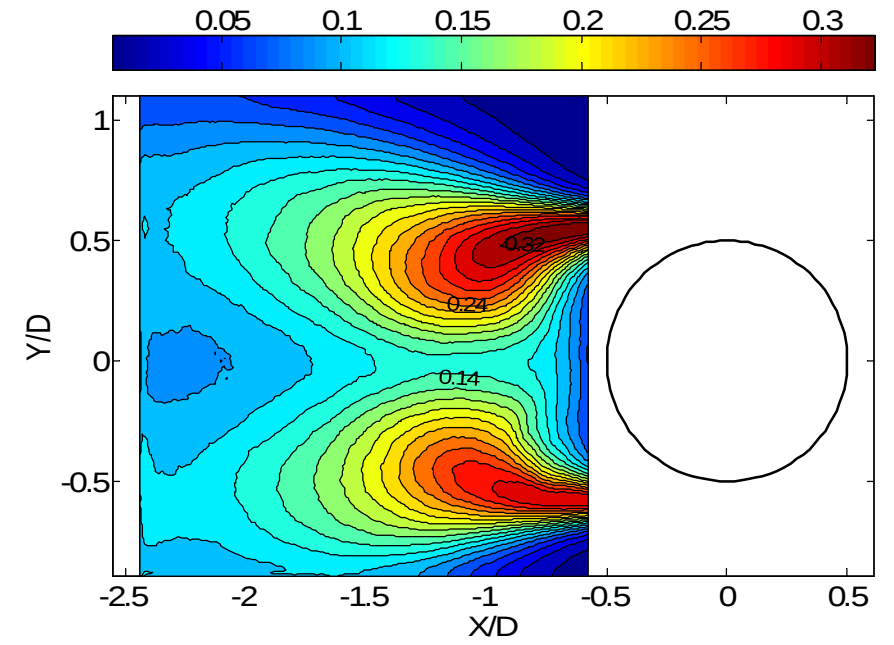

Figure 7 Flow pattern I : spatial field of $\mathrm{u}^{2} / U$ in the wake of the upstream cylinder.

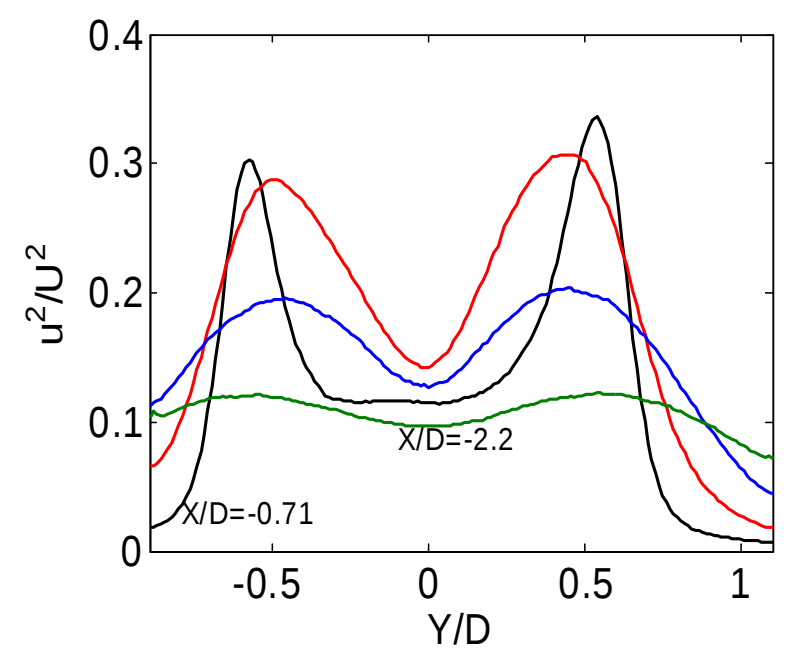

Figure 9 Flow pattern I: profiles of $\dot{u}^{2} / U$ along the lines $\quad x / D=-0,71$ (black) $\quad x / D=-1 \quad$ (red) $x / D=-1,5$ (blue) $x / D=-2,2 \quad$ (green).

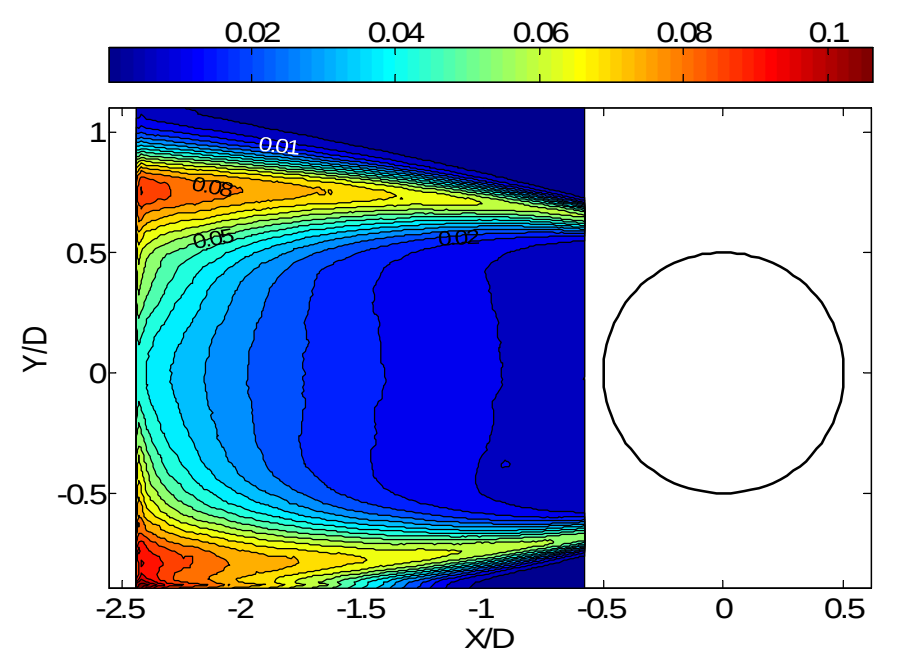

Figure 8 Flow pattern II : spatial field of $\dot{u}^{2} / U$ in the wake of the upstream cylinder.

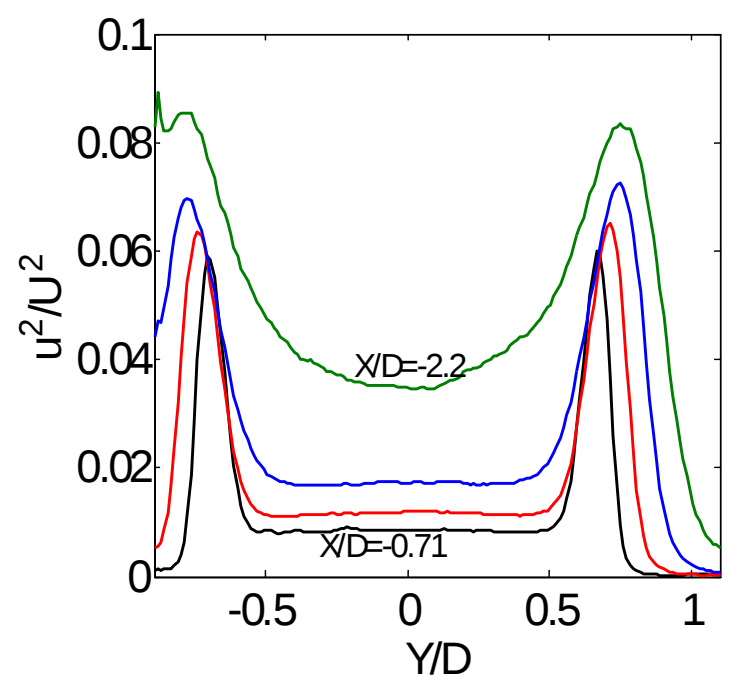

Figure 10 Flow pattern II : profiles of $\quad^{\prime} / U$ along the lines $x / D=-0,71$ (black) $x / D=-1 \quad$ (red) $x / D=-1,5$ (blue) $x / D=-2,2 \quad$ (green). 


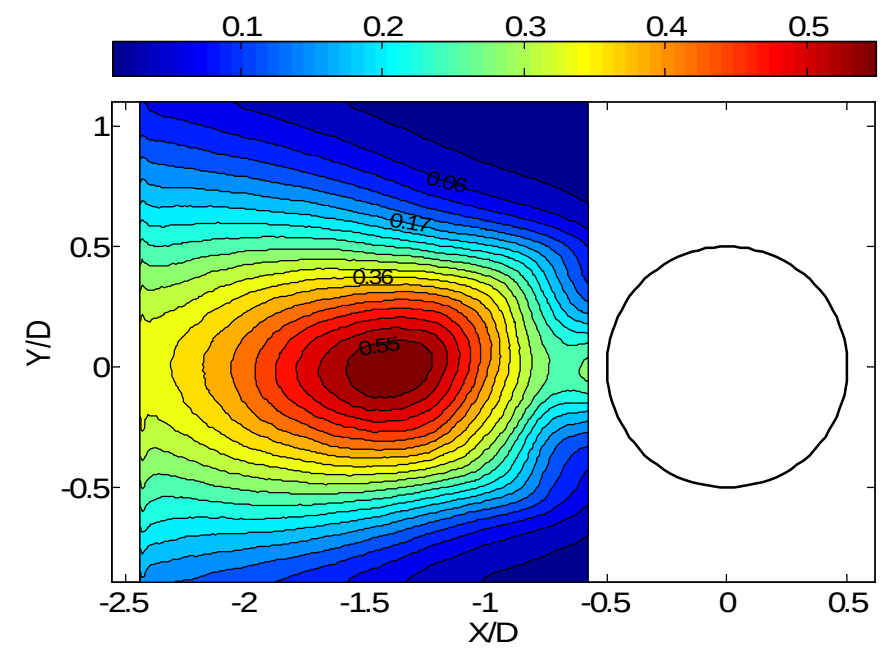

Figure 11 Flow pattern I: spatial field of $v^{\prime} / U$ in the wake of the upstream cylinder.

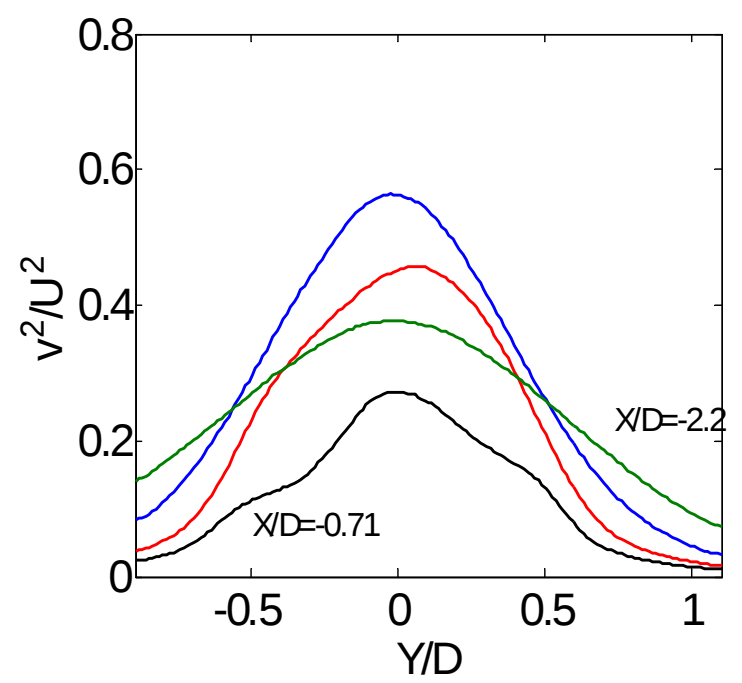

Figure 13 Flow pattern I : profiles of $v^{2} / U$ along the lines $\quad x / D=-0,71$ (black) $\quad x / D=-1$ $x / D=-1,5$ (blue) $x / D=-2,2 \quad$ (green).

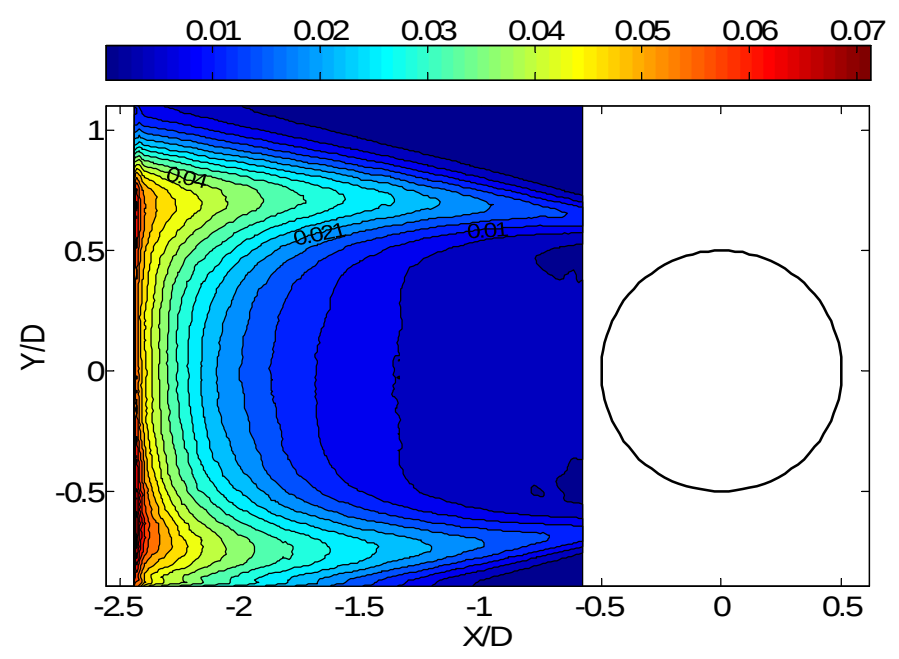

Figure 12 Flow pattern II : spatial field of $v^{\prime} / U$ in the wake of the upstream cylinder.

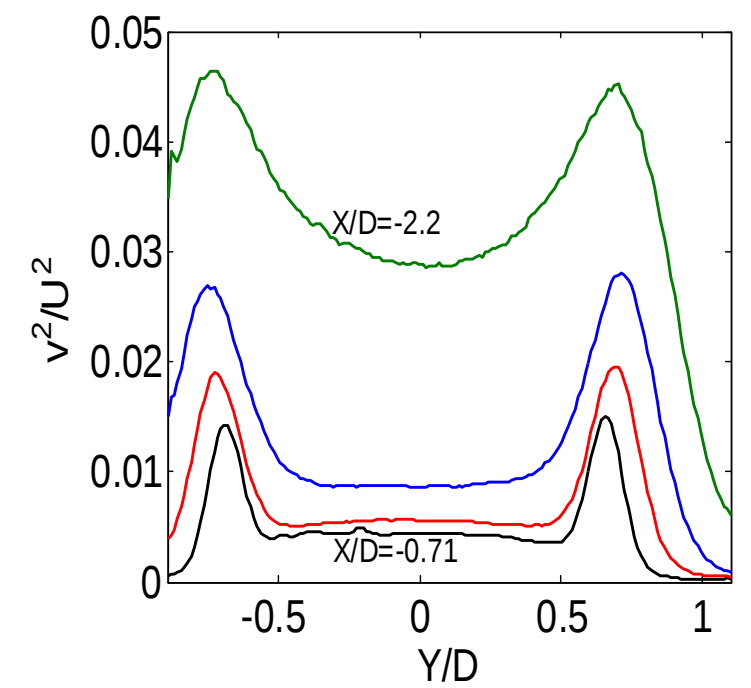

Figure 14 Flow pattern II: profiles of $v^{\prime} / U$ along the lines $x / D=-0,71$ (black) $x / D=-1$ (red) $x / D=-1,5$ (blue) $x / D=-2,2 \quad$ (green). 


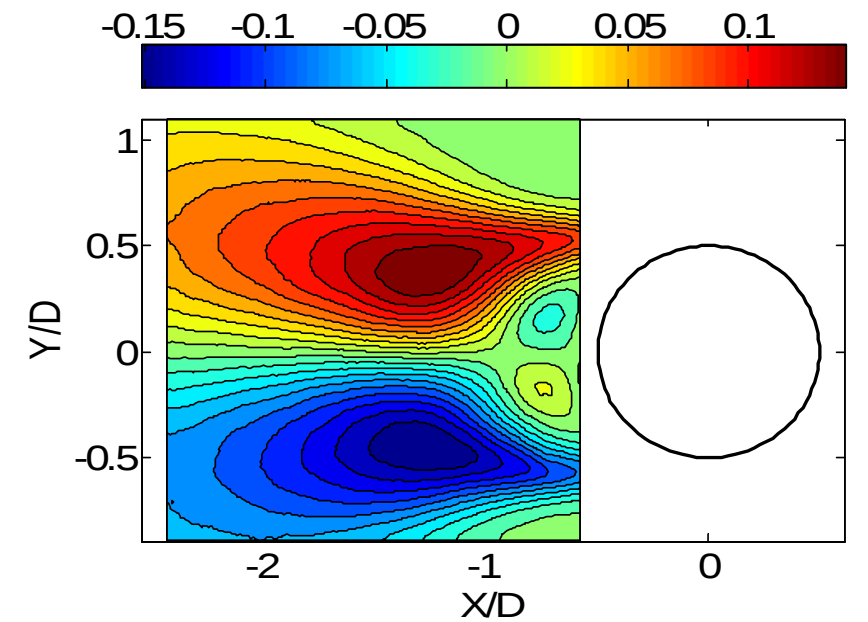

Figure 15 Flow pattern I : spatial field of $u$ vi $U$ in the wake of the upstream cylinder.

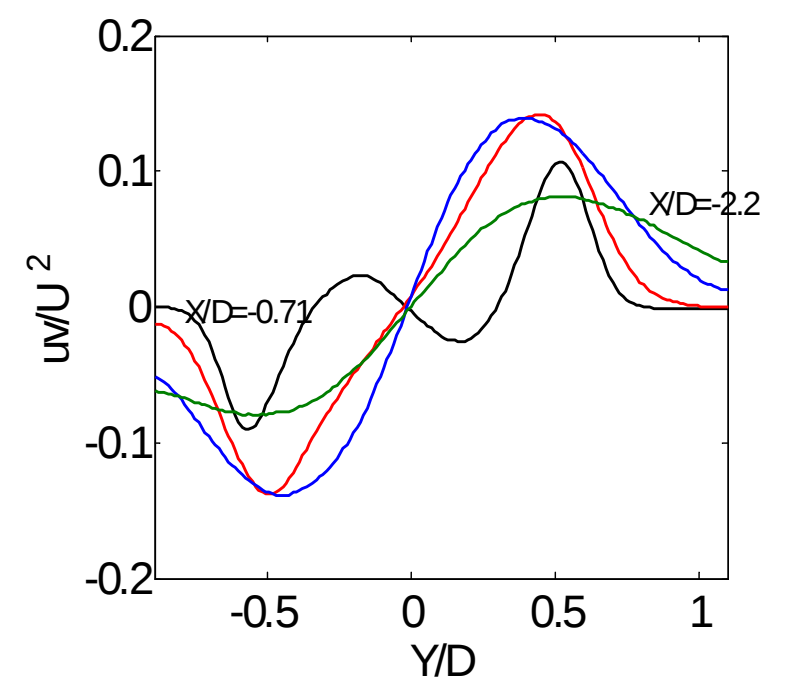

Figure 17 Flow pattern I : profiles of $u ́ v / U$ along the lines $\quad x / D=-0,71 \quad$ (black) $\quad x / D=-1 \quad$ (red) $x / D=-1,5 \quad$ (blue) $x / D=-2,2$ (green).

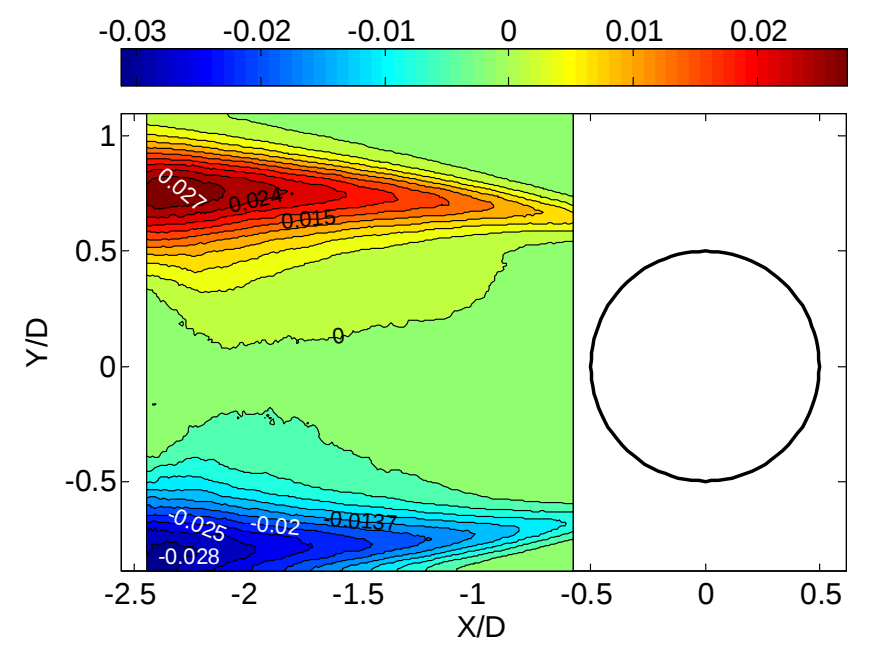

Figure 16 Flow pattern II: spatial field of $u$ v $/ U$ in the wake of the upstream cylinder.

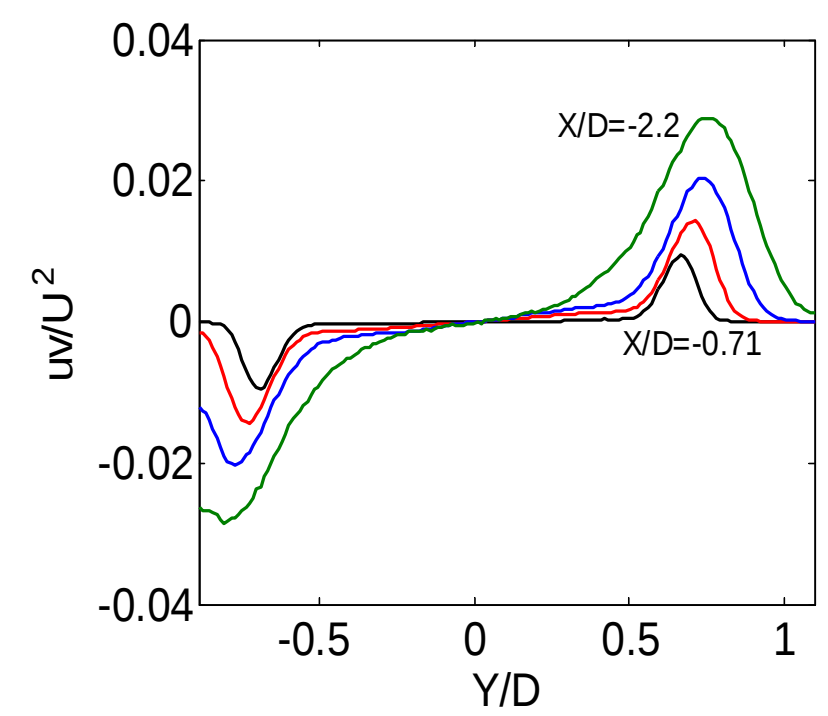

Figure 18 Flow pattern II : profiles of úv/U along the lines $x / D=-0,71$ (black) $x / D=-1 \quad$ (red) $x / D=-1,5 \quad$ (blue) $x / D=-2,2 \quad$ (green). 


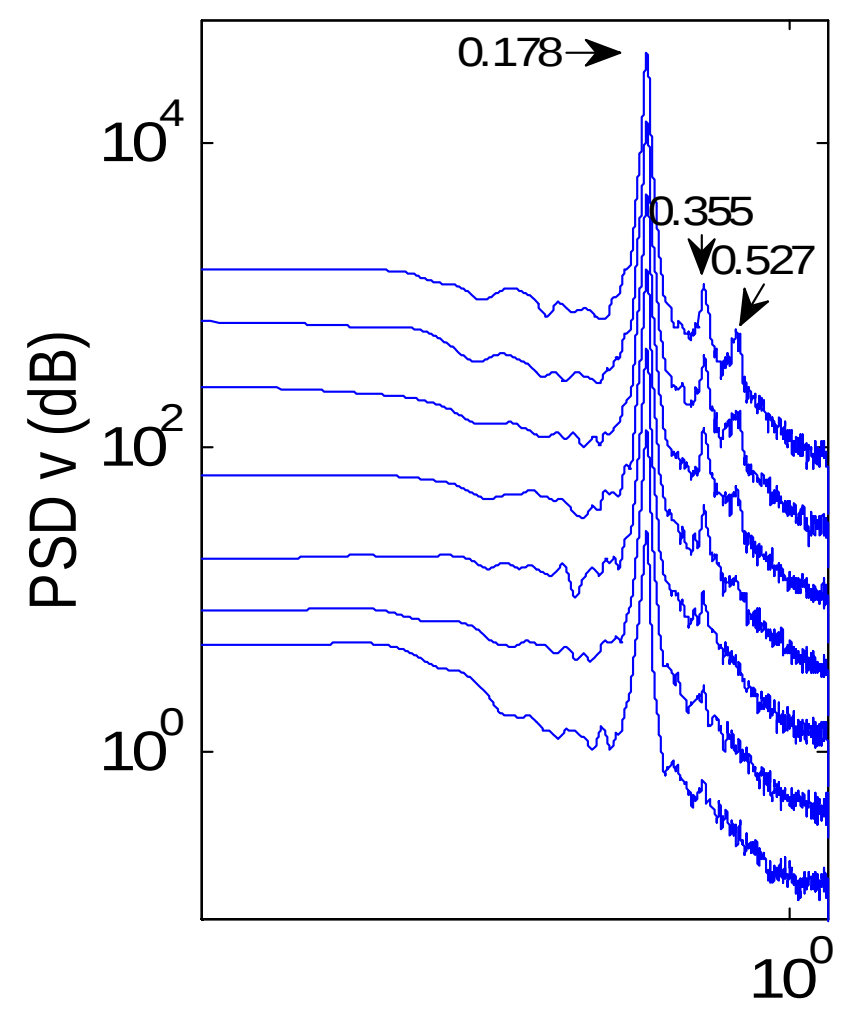

f.D/U

Figure 19 Flow pattern I : vertical velocity periodogram on the horizontal line $y / D=0,5$ at positions from $x / D=-0,76$ to $x / D=-2,17$ by a step of $-0,2344$

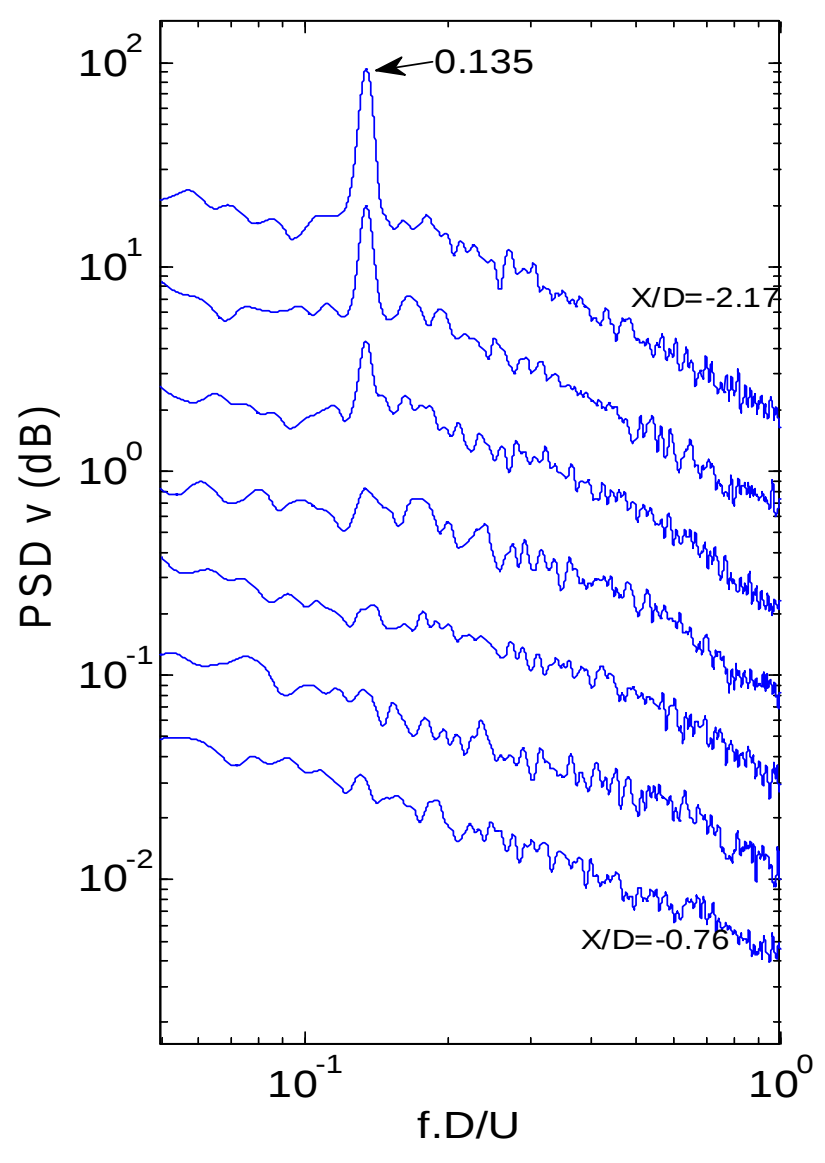

Figure 20 Flow pattern II : vertical velocity periodogram on the horizontal line $y / D=0,5$ at positions from $x / D=-0,76$ to $x / D=-2,17$ by a step of 